\title{
Hard Coating Deposits: Incompatible Working Energy and Forced Behaviors of Gaseous and Solid Atoms
}

\author{
Mubarak Ali $^{\text {a }}$, Esah Hamzah ${ }^{b}$, Mohd Radzi Mohd Toff c \\ a Department of Physics, COMSATS University Islamabad, Park Road, Islamabad - \\ 45550, PAKISTAN, ”E-mail: mubarak74@mail.com, mubarak74@comsats.edu.pk \\ b Department of Materials, Manufacturing and Industrial Engineering, Faculty of \\ Mechanical Engineering, Universiti Teknologi Malaysia, 81310 Skudai, Johor, \\ MALAYSIA, E-mail: esah@fkm.utm.my
}

c Advanced Materials Research Center (AMREC), SIRIM Berhad, Lot 34, Jalan Hi-Tech 2/3, Kulim Hi-Tech Park, 09000 Kulim, Kedah, MALAYSIA, E-mail: mradzit@sirim.my

Abstract: Coating a suitable material on substrate in thickness of nano to micro metres is a great interest for the scientific community. Hard coatings develop under the significant composition of suitable gaseous and solid atoms, where their energy and forced behaviors under certain transition states favour the binding. In the binding mechanism of gaseous and solid atoms, electron belonging to outer ring (filled state) of gaseous atom undertakes another clamp of energy knot belonging to outer ring (unfilled state) of solid atom. The set process conditions develop the coating of gaseous and solid atoms when energy of non-conservation is involved. Different natured atoms develop the structure in the form of hard coating by locating a common ground point, which is in their central ground points. Here, gaseous atoms increase the potential energy of electrons by decreasing levitational force (at electron level) in a controlled orientating manner, whereas solid atoms decrease the potential energy of electrons by decreasing gravitational force (at electron level) in a controlled orientating manner. Thus, hard coating is deposited under the oppositely switched energy and forced behaviors of different natured atoms. In TiN coating, Ti-Ti atoms bind due to the difference of expansion in their lattices when one atom is deposited and one is being 
deposited. So, one $\mathrm{Ti}$ atom just lands on the already landed $\mathrm{Ti}$ atom. While adhering $\mathrm{N}$ atom to $\mathrm{Ti}$ atom, it occupies the interstitial position in the $\mathrm{Ti}$ atoms. The rate of ejecting solid atoms depends on the type of source, parameters and a processing technique. In random arc-based vapor deposition system, depositing coating at substrate depends on several parameters. As per set conditions of the process, different natured atoms deposit at substrate surface to develop the structure of coating. In addition to intrinsic behavior of atoms, different properties of coatings materialised as per the nature of forces engaged under the involved energy. In developing hard coating of gaseous and solid atoms, an involved energy of non-conservation engages force of nonconservation, too. Hence, this study opens new avenues not only in the fields of hard coatings but also in the fields of functional coatings, medical and surgical implant coatings, protective and sensitive coatings, etc.

Keywords: Fundamental science; Atomic behavior; Hard coating; Expansion and contraction; Energy and force; Surface and interface

\section{Introduction}

Hard coatings are an integral part of scientific research and technological advances. In the market, hard coatings for different purposes are in routine use, where their composition and deposition techniques are debated thoroughly. In this context, several materials comprising different composition of atoms are available underlining their deposition history. They highlight features of deposited coatings through their surface and interface study. To develop coatings of different characteristics, a variety of deposition techniques are available. In coatings, a minute quantity of deposited materials over less-important or not practically viable material gives the value-added results.

Ultra-precision machine tool coatings, coatings of medical implants and surgical instruments, transparent and insulating coatings, decorative and protective coatings and coatings for other miscellaneous uses are in the routine demand. A variety of techniques are involved in depositing different sorts of coatings. Coatings are mainly used for two reasons - first, for the potential use of the coated part, and second, for the 
substitution of any desired composition of atoms. Coating the surface of suitable substrate results in its different functioning.

Atoms do not ionize while atoms of inert behavior split under the excessive population of photons having characteristics of current, as explored by Ali [1]. A silicon atom having neutral behaviour electrons converts heat energy into photon energy, as clarified by Ali [2]. Solid atoms belonging to certain elements evolve structures of different dimension and format as per built-in inter-state electron gap, where conservative forces are involved to execute confined inter-state electron dynamics [3]. The origin of atoms of some elements in gaseous state and some elements in solid state has been discussed [4]. A gaseous carbon atom originates several different states due to the involvement of typical energy and thereby, provides the path for filled state electron to migrate to nearby unfilled state [5]. Solid atoms under different modifications can develop tiny-sized particles of different impact in nanomedicine [6].

The prosperous assembling of colloidal matter into meaningful structure treats atoms and molecules as future materials and it has been discussed by Glotzer and Solomon [7]. The understanding of the individual dynamics of the formation of tiny particles is essential before assembling them into the useful large-sized particles [8]. The origin of physics and chemistry of materials through the formation process of tiny particles and their extended shape particles in pulse-based electron-photon/solution interface process was discussed by Ali and Lin [9]. Ali et al. [10] discussed the processing of gold and silver solutions at nearly identical conditions in the same method, where geometric structures developed only in the case of gold. When the atoms were in suitable transition state under controlled conditions of process, gold particles of geometrical shapes developed [11].

Different behavior of 'tiny grain carbon films' in Raman spectroscopy and energy loss spectroscopy analyses were studied by Ali and Lin [12]. Switching morphological structure of grains and crystallites under slightly altered parameters in developing carbon films was discussed by Ali and Ürgen [13]. Under varying chamber pressure, a discernible change in the morphology and the growth rate of carbon films was observed by Ali and Ürgen [14]. These studies indicated a very different behavior of a carbon 
atom despite having the same number of electrons. Clearly, these studies provide the basic understanding of atoms of all elements. The present study concentrates on the deposition of hard coating by arc-based technology.

Some earlier studies have reported the history of arc-based technology along with the initial efforts of deposition of suitable materials [15-18]. The basic engineering of d.c. arc discharge for different cathodes was investigated by Wroe [15]. The application of vacuum arc to deposit thin films and coatings was documented in a book written by Boxman et al. [16]. The advantages and disadvantages of different cathodic arc sources were studied by Karpov [17]. In deposition of films through cathodic arc, the presence of macroparticles was considered to be disadvantageous for some applications as discussed by Brown [18].

In depositing TiN coatings on different substrates under varying process conditions employing cathodic arc physical vapor deposition, different morphology, structure, hardness, surface roughness, friction coefficient, adhesive strength and performance of coated tools were reported [19-25]. The deposition of TiN coatings with different $\mathrm{N}$ gas flow rates has been studied by Ali et al. [19]. The effects of different $\mathrm{N}$ gas flow rates on the friction coefficient and surface roughness of TiN coatings have been discussed by Hamzah et al. [20]. Cathodic arc vapor deposition to optimize the thickness of TiN coating deposited on different substrates was presented by Ali et al. [21]. The evaluation of friction coefficient and surface roughness as a function of temperature in different deposited TiN coatings was studied by Ali et al. [22]. The generation of macrodroplets at different parameters depositing TiN was investigated [23]. The surface roughness of TiN coatings deposited on steel substrate under several parameters was investigated by Ali et al. [24]. A way to improve adhesiveness of TiN coating to substrate along with the growth mechanism of macrodroplets was studied by Ali et al. [25].

There are several studies that discuss TiN coatings, processing techniques and analyses [26-33]. A droplet-free TiN coating by an improved and modified vapor deposition technique was deposited by Elmkhah et al. [26]. The properties of TiN coating for targeted application were improved through the bombardment of active 
species by Oliveiraa et al. [27]. TiN coated as an interlayer material along with TaN was prepared and their science along with the application was elaborated by Vogel et al. [28]. Droplet-related defects produced through cathodic arc vapor deposition have important implications both for science and technology [29]. TiN coating on stainless steel substrates to study tribological features under different conditions and was deposited by Bahri et al. [30]. A review on the improvement of adhesive properties of TiN coatings was presented by Othman et al. [31]. In suitable morphology, TiN as an electrode material for dye-sensitized solar cell was studied by Jiang et al. [32]. Macroparticle content in cathodic arc deposited TiN coatings by varying the position of target was reported by Harris et al. [33].

Different types of hard coatings developed under various conditions have also been published extensively [34-45]. The hardness of Ti (C, N) was significantly improved under the control of microstructure [34]. To achieve firmness in two materials and protection of the coating adherence to tool against abrasion were studied by Bobzin [35]. Tribological properties under wet conditions were studied for different hard coatings including TiN coating [36]. A theoretical study for hard coating was presented by Music et al. [37], where both DLC and TiAIN based coatings were studied in a new direction. A review on the features of $\mathrm{TiN}$-based coatings related to wearable resistance was studied by Santecchiaa et al. [38]. Investigation of hard and tough features of some important ceramic coatings along with the underpinning mechanism for the better future of spray technique were recommended [39]. The composition of deposited coatings and their achieved morphologies remained central to advance hard coatings for various cutting tool applications as discussed by Inspektor and Salvador [40]. A review on multicomponent, nanocomposite, and protective coatings was presented by Pogrebnjaka et al. [41]. A review on the corrosion behavior of hard coatings for different steel substrates was investigated by Fenker et al. [42]; microstructure was expected to play the secondary role only for corrosion behaviour of coating. Zirconium nitride coatings produced by cathodic arc deposition technique and high-power impulse magnetron sputtering were compared by Purandarea et al. [43]. Different hard coatings deposited on hot-working tool steel in the practical die casting service were evaluated 
by Mitterer et al. [44]. A comparison of TiAIN coatings in different vapor deposition techniques was presented by Münz et al. [45].

In addition to depositing hard coatings for cutting tools' application, they are also in use for other applications. The suspension of hard coating to improve the scratch resistance of PMMA surface was studied by Tanglumlert et al. [46]. The effects of different parameters in depositing nanocomposite hard coatings for solar thermal applications were discussed by Soni et al. [47]. Increasing sputtering power upto a certain extent improved the deposition rate of film [48]. Hard features in the nano-hybrid coatings depositing over the polymeric substrate were explored by Eshaghi [49]. Fracture toughness of $\mathrm{Ti}_{1-x} \mathrm{Zr} \times \mathrm{N}$ hard coatings in relation to the elemental composition by using the internal energy induced cracking method was studied by Huang et al. [50]. According to Siow et al. [51], the properties of TiCN coating are controlled under the maintenance of $\mathrm{C}$ and $\mathrm{N}$ ratio depositing on the tungsten carbide. The significant properties of TiN coatings on Ti substrate for implanting applications were investigated by Uddin et al. [52]. Different coating methods with relevance to metallic implants were categorized and investigated where comparative study of the techniques was also presented [53]. Combining points of different deposition techniques to enable the deposited coating deliver maximum performance can be the next target of scientific community [54].

The basic idea discussed in the studies mentioned above is related to the properties (and characteristics) of deposited coatings, which are mainly subjected to the change in the process parameters, types of material used and the processing approach. Here, it is discussed that hard coating is deposited due to incompatible working energy and forced behaviors of gaseous and solid atoms.

In addition to the scientific details available for hard coatings discussed above, coatings are a way to express relation between the comprised atoms. This study reports emphasized the fundamental aspects of developing hard coatings depositing TiN coating on a high-speed steel (HSS) disc employing random arc-based vapor deposition technique. This work presents the fundamental aspects of depositing different hard coatings in general and investigating mechanism of developing TiN coating in particular. 


\section{Experimental details}

HSS discs were utilized as a substrate material for the deposition of TiN employing the commercially available coating unit known as 'cathodic arc physical vapor deposition technique', which is now termed as 'random arc-based vapor deposition'. After the required cleaning, the samples having diameter such as $10 \mathrm{~mm}$ and thickness such as $6 \mathrm{~mm}$ were loaded in the coating system (Hauzer Techno Coating (HTC) 625/2 ARC). The complete deposition procedure along with the metallographic process of samples has been described in the earlier work of the same group [25]. Surface topography and interface cross-sectional view were captured by using field emission scanning microscope (FE-SM also known as FE-SEM, Model LEO-1525). The thickness of the deposited coatings was measured under the application of FE-SM and through the captured fractured cross-sectional image of the deposited coating on HSS substrate. Prior to coating TiN on HSS disc, an inter-layer of Ti atoms was deposited for the 15 minute process. The purpose of depositing inter-layer was to enhance the adhesive strength of the following TiN coating. Thus instead of $\mathrm{N}$ gas, first an inert Ar gas was regulated through mass flow controller to ignite the arc. At the start of depositing interlayer, chamber pressure was $5 \times 10^{-6} \mathrm{mbar}$. While depositing inter-layer, $50 \mathrm{sccm} \mathrm{N}$ gas flow rate was maintained by mass flow controller meter. To deposit TiN in the form of coating, substrate temperature was maintained at $300^{\circ} \mathrm{C}$, where $\mathrm{N}$ gas flow rate was $250 \mathrm{sccm}$. The bias voltage was 50 volts and rotational speed of the substrate holder was controlled to deposit coatings of certain features [25]. Input current for igniting arc to eject $\mathrm{Ti}$ atoms from the target was $100 \mathrm{~A}$. Total duration of the deposition process was set at $90 \mathrm{~min}$.

\section{Results and Discussion}

Figure 1 (a) shows surface topography of the deposited TiN coating on HSS disc, where the surface is partially covered with macrodroplets (MDs) of a few hundred of nanometers to a few microns in size. The distribution of MDs is uniform throughout the surface of deposited coating. A large sized MD in the central vicinity of deposited coating is displayed in Figure 1 (a), which also shows mapping of the region, where the 
concentration of both $\mathrm{Ti}$ and $\mathrm{N}$ atoms is in different colors. Figure 1 (b) reveals fractured cross-sectional view of the coating, where initially deposited $\mathrm{Ti}$ inter-layer shows thickness less than one micron. Atoms of $\mathrm{Ti}$ inter-layer adhere to substrate under favorable conditions; a different texture of a few nanometers deposited layer (between the substrate and deposited TiN coating) can be observed in Figure 1 (b).
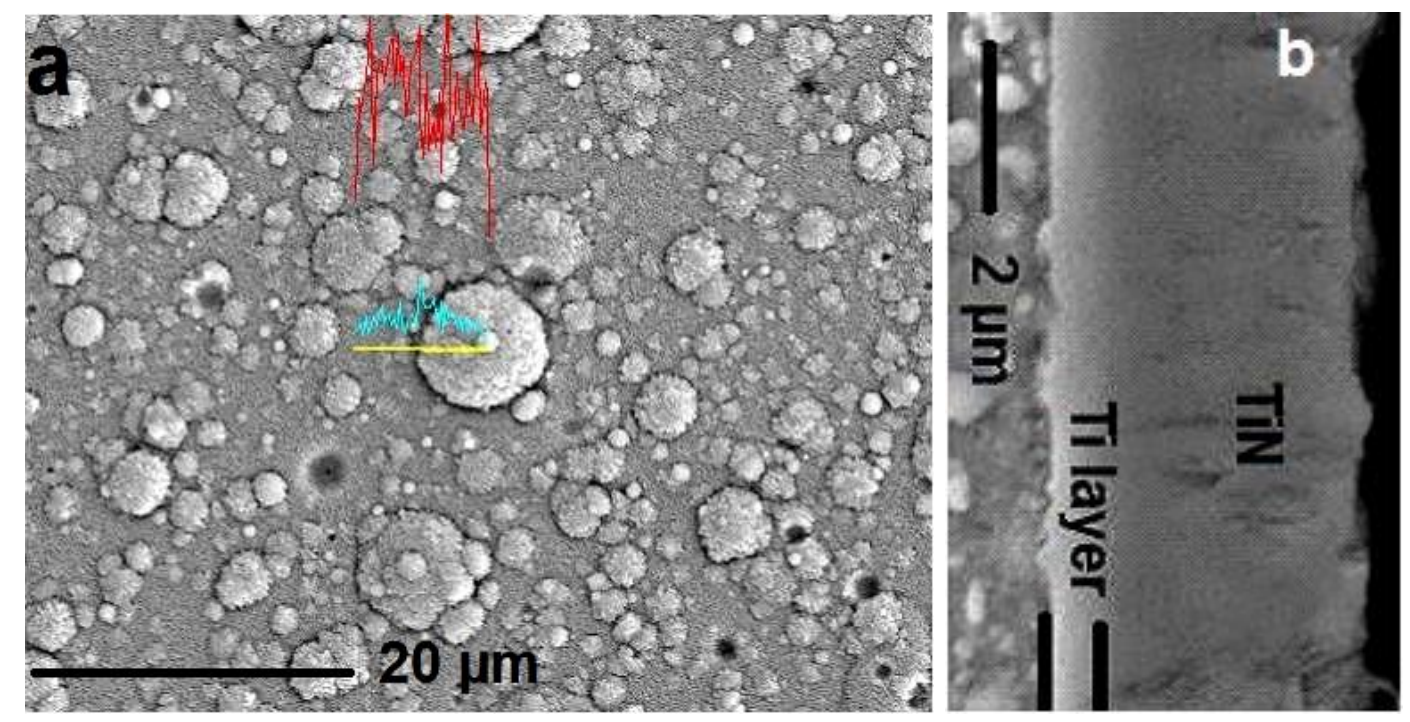

Figure 1: (a) topographic view of TiN coating on HSS disc and (b) a few hundred nanometers thick Ti inter-layer shows contrast with respect to afterward deposited TiN coating of thickness $\sim 4 \mu \mathrm{m}$

Substrate surface comprising different elements like W, Mo, Cr, V, C and Fe adhering $\mathrm{Ti}$ atoms at initial stage improves the adhesive strength of the afterward deposited coating [19, 20]. Ti atoms bind to the substrate surface under suitable conditions of the deposited inter-layer. The substrate surface that consists of atoms of different elements is required to deposit $\mathrm{Ti}$ atoms up to a few nanometers' thickness. This inter-layer is required to improve the adhesive strength of the afterward deposited coatings. Some preliminary details regarding the adhesive strength of TiN coating deposited under different conditions in random arc-based vapor deposition system are discussed by Ali et al. [25].

Figure 2 exhibits the mapping of Ti atoms in the deposited TiN coating at the point of MD (in Figure 1a). The content of $\mathrm{Ti}$ atoms is around $70 \%$, which is shown in the form of histogram. This indicates that MD contained less concentration of $\mathrm{N}$ atoms. This also indicates that the coating can also contained $70 \%$ Ti content. 


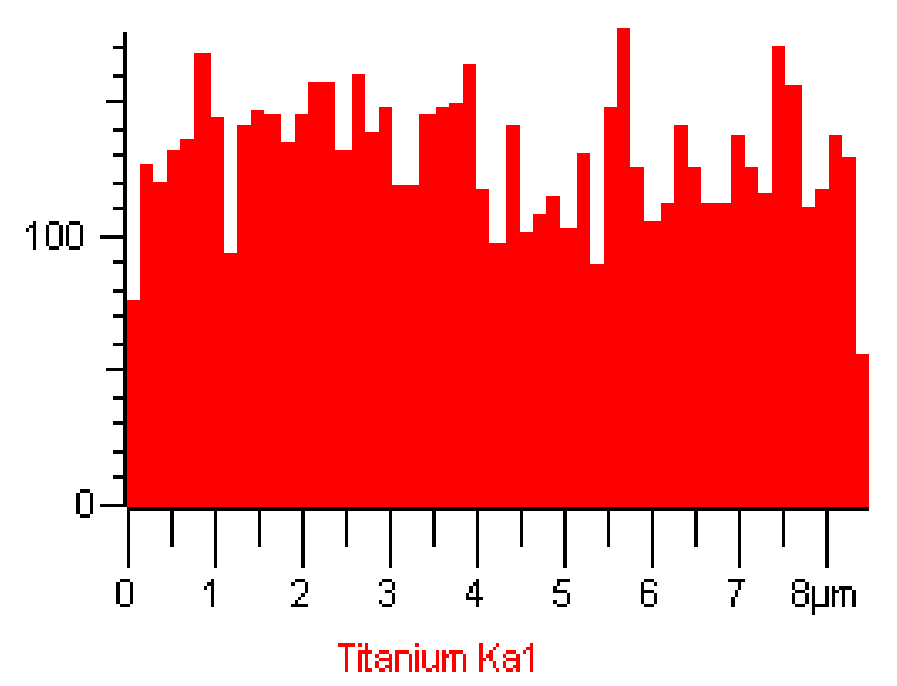

Figure 2: Mapping of Ti atoms in terms of content ratio distribution in TiN coating

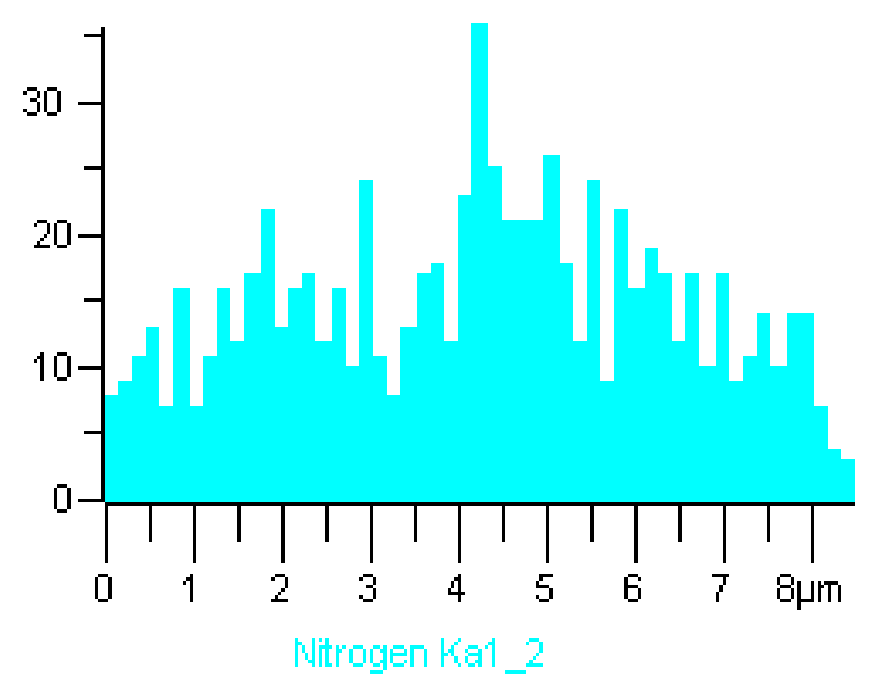

Figure 3: Mapping of $N$ atoms in terms of content ratio distribution in TiN coating

Figure 3 shows the mapping of $\mathrm{N}$ content in TiN coating in the form of histogram, where $\mathrm{N}$ atoms is around $30 \%$ at the central point of MD displayed in Figure 1 (a). This indicates that the $\mathrm{N}$ atoms in the coating can also contain $30 \%$ of content. The distribution of $\mathrm{N}$ atoms in the coating is also uniform. There are several studies, where the reduction of MDs in depositing hard coatings was investigated under the different process conditions [19, 21-23].

A hard coating belongs to the category of refractory materials. As this type of material does not conduct field despite the fact that it contains a major component of metallic nature atoms. About $70 \% \mathrm{Ti}$ atoms in the coating is found as evident in Figure 
2. Adherence of gaseous atoms to solid atoms develops a hard coating showing its insulator behavior. A photonic field propagating through the irregular inter-state electron gaps of hard coating structure is disturbed. The incorporated gaseous atoms build the bridges via electrons in bound atoms of conductive behavior. So, photons having characteristics of current deal with disruption at the input end of applied field. A detailed study presented by Ali [1] discussing the significance of inter-state electron gaps while propagating photons; it is further discussed that atoms of none of the elements have band gap between conduction band and valence band. In a structure, an overall configuration of inter-state electron gap determines the strength of propagating photonic field. This indicates that a science of semiconductors requires new explanation.

In random arc-based vapor deposition system, atoms of Ti or other types of metallic elements are ejected from the targets. Under the supply of high power propagating field, an arc in random shape ejects atoms. At the high concentration of $\mathrm{N}$ atoms, a random arc is steered to eject $\mathrm{Ti}$ atoms both in atomic form and droplet form. Depending on the nature of source of metallic atoms and employed conditions of the process, the properties of resulted coating are varied. The basic layout of ejecting $\mathrm{Ti}$ atoms and entering $\mathrm{N}$ atoms to deposit TiN coating is sketched in estimation as shown in Figure 4.

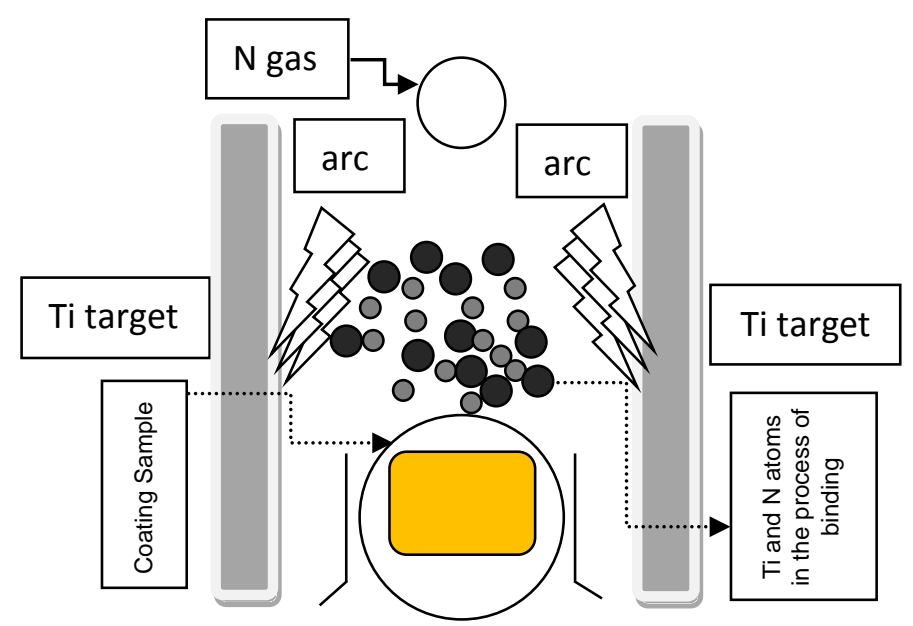

Figure 4: A layout of depositing Ti atoms and $N$ atoms to develop TiN coating at HSS substrate The newly observed atomic structures of $\mathrm{Ti}$ and $\mathrm{N}$ elements are sketched in Figure 5 (a) and (b). The tiniest particles known as electrons are filled in the hollow spaces designed by the particular scheme of intercrossed overt photons. Each hollow space is 
covered by energy knot, which forms a filled or unfilled state. Depending on the number of filled and unfilled states, the energy knots forming the lattice of an atom varied [4]; filled and unfilled states obey a configuration of their atom designated by its element. A study conducted by Ali [5] constructed the lattice (energy knot net) of carbon atom.

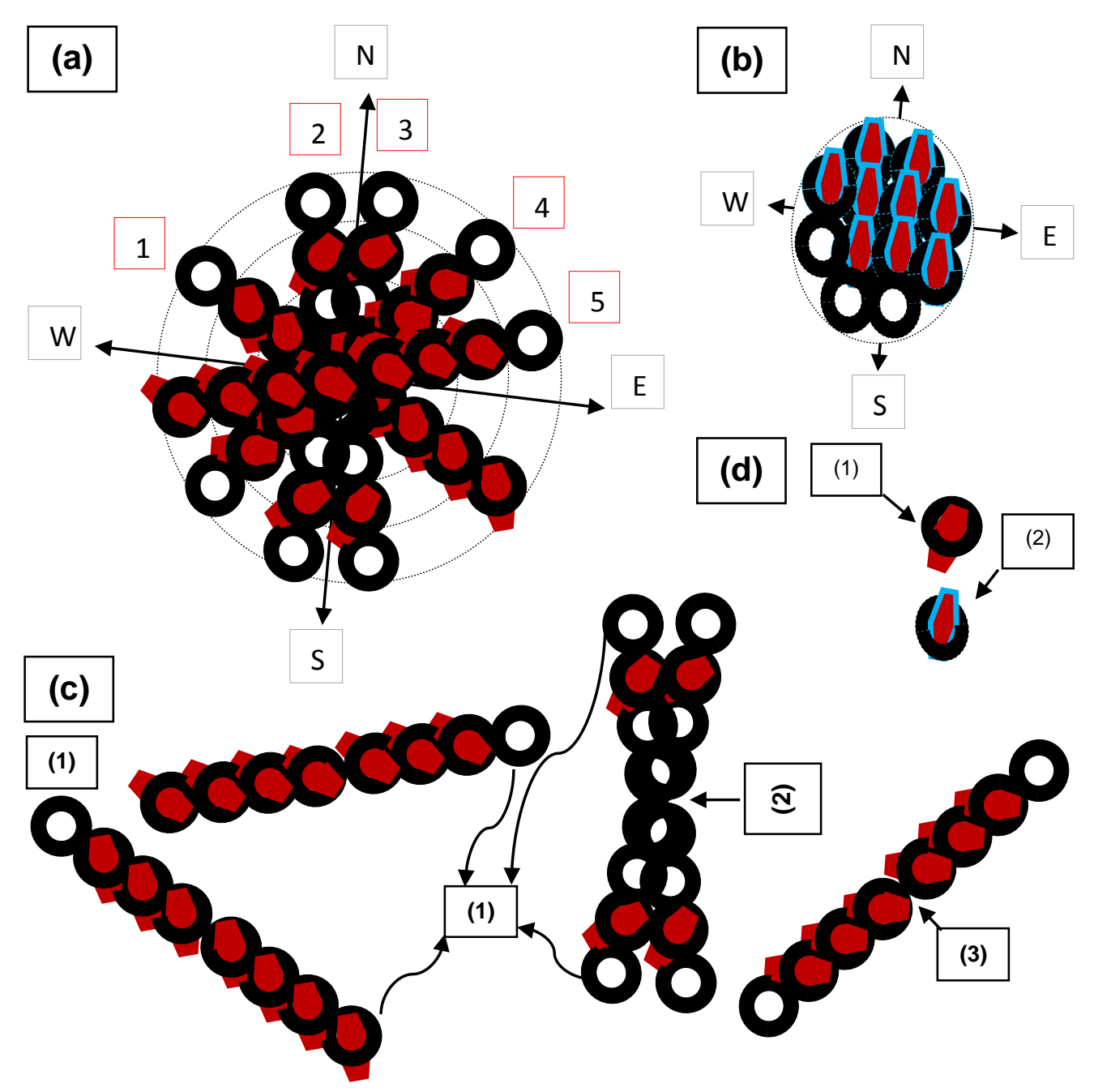

Figure 5: Atomic structure of (a) Ti atom (4 electrons in zeroth ring, 8 electrons in $1^{\text {st }}$ ring, 10 electrons in $2^{\text {nd }}$ ring and 2 electrons along with 8 unfilled states in $3^{\text {rd }}$ (outer) ring), (b) $N$ atom (4 electrons in zeroth ring, 5 electrons and 3 unfilled states in $1^{\text {st }}$ ring (outer) ring), (c) chains of filled and unfilled states along with folded states in Ti atom; (1) unfilled and filled states at the ends of chains, (2) inner unfilled states are folded by filled states and (3) a chain of states having inner states filled and outer states of both ends unfilled and (d) a single electron of Ti atom and $N$ atom is shown where (1) electron clamped by expanded energy knot under increased potential energy in Ti atom and (2) electron clamped by contracted energy knot under decreased potential energy in $N$ atom 
For $\mathrm{Ti}$ atom, total 32 states of electrons are available, but 24 states are filled by the electrons while 8 states remained unfilled. Both filled and unfilled states of $\mathrm{Ti}$ atom are constructed by the intercrossing of overt photons having length measured in required number of states. The unfilled states belonging to outer ring in $\mathrm{Ti}$ atom are shown in Figure $5(a)$.

In the case of inner unfilled states of $\mathrm{Ti}$ atom, they are folded by the neighbouring filled states as indicated in (2) of Figure 5 (c); these are not counted in atoms (of any element) to define filled and unfilled states. Electrons, which do not occupy the position at the ends of some chains in $\mathrm{Ti}$ atom, are shown separately in Figure 5 (c). So, chains in $\mathrm{Ti}$ atom keep both filled states and unfilled states. In $\mathrm{Ti}$ atom, there are total five chains of states as separately shown in Figure 5 (c). A chain of states in any atom is constructed by the intercrossing of oppositely directed two overt photons. In case of $\mathrm{Ti}$ atom, each overt photon contains 8 'unit photons'. Two overt photons of opposite side when intercrossed by keeping the centre lengths at common point, they form (or construct) a chain of states in $\mathrm{Ti}$ atom. Hence, overt photons comprising length of eight 'unit photons' intercross to form a chain of filled and unfilled states of electrons in each case. As shown in Figure 5 (c), five such shapes are drawn; their precise intercrossing from the center lengths form the 'energy knot net' of Ti atom. In this way, 24 filled states and 8 unfilled states are formed.

Energy knots clamping electrons in $\mathrm{Ti}$ atom and $\mathrm{N}$ atom are symbolically displayed in Figure 5 (d); in electron of Ti atom, clamped energy knot expanded as shown in (1) and in electron of $\mathrm{N}$ atom, clamped energy contracted as shown in (2).

Filled states of outer ring in the atoms of solid and gaseous states are donated by the positive valency and negative valency, respectively. In the case of $\mathrm{Ti}$ atom, valency is +2 , so it has ' 8 ' unfilled states. In the case of $N$ atom, valency is -3 , so it has ' 5 ' unfilled states. Hence, negative sign of valency in gaseous atoms infers that their ground points are above the suitable level of ground surface and positive sign of valency in solid atoms infers that their ground points are below the suitable level of ground surface. 
In different coating units, regardless of the required numbers of atoms per unit area or volume, they are deposited under set parameters of the process, and their involved energy is based on individually attained dynamics plus electron dynamics. Hence, input power is the key to regulate the structure of atoms. So, different characteristics of their coating are materialised. A developing structure of TiN is within the short-range order. So, a TiN coating is developed having a non-homogeneity in overall structure.

Each $\mathrm{Ti}$ atom occupies two electrons in its outer ring. This low number of filled states enables it to occupy many unfilled states in the outer ring. Being a solid atom, it keeps unfilled states above the east and west poles both on left and right sides of the northpole. As the Ti belongs to solid element, so electrons of filled states in outer ring of atom remain below the east and west poles both on left and right sides of the south-pole (in Figure 5a). On the other hand, five filled states in the outer ring of $\mathrm{N}$ atom allow a smaller number of unfilled states in that ring. Due to the gaseous nature of $\mathrm{N}$ atom, it contains several filled states of outer ring, where majority of the electrons (filled states) are expected to be above the east and west poles both on left and right sides of the north-pole (in Figure 5b). The availability of several unfilled states of outer ring in $\mathrm{Ti}$ atom offers a provision for suitable unfilled state to have an affinity to suitable electron of filled state of outer ring in $\mathrm{N}$ atom when suitable conditions of the process are being provided. Hence, a suitable electron of gaseous atom is being clamped by unfilled energy knot of solid atom. In other words, binding of gaseous atom to solid atom is through double clamping of energy knot to electron.

When gaseous atoms are in the re-crystallization state, their electrons move downward under infinitesimal displacements, where they decrease levitational force by increasing their potential energy. When solid atoms are in the re-crystallization state, their electrons move upward under infinitesimal displacements, where they decrease the gravitational force by decreasing the potential energy. When gaseous and solid atoms attain the suitable transition states, the electron of a gaseous atom experiences force to north-sided tip through hollow space of unfilled energy knot of solid atom. Thus, that electron undertakes another clamp of energy knot in addition to its own clamp. This mechanism of double clamping of electron by unfilled energy knot is under the suitable 
transition states of atoms. When many different atoms of such type follow the same scheme, they develop hard features of their coating.

Atoms of metallic targets are in their processed form as they are extracted from the ore. It is expected that they are with their original state behaviour, and so is the case with electrons and energy knots of atoms. On the other hand, entered gaseous atoms to the chamber are also in their processed form. Again, it is expected that they are with their original state, and so is the case of electrons and energy knots of atoms. Under applied conditions of the process and just at the stage of recovering original state, they bind under suitable coinciding, where electron of gaseous atom undertakes another clamp of unfilled energy knot of solid atom. Therefore, solid atoms have already carried out their work negatively while coming near the suitable level of ground surface from the south side, whereas gaseous atoms have already worked positively while coming near the suitable level of ground surface from the north side. To recover the original state behaviours of two different natured atoms, they work for different energy and forced behaviours where the job done by the gaseous atom will be (just) negative, while the job done by the solid atom will be (just) positive.

In depositing different natured atoms in a vapor deposition system, electrons of outer rings belonging to gaseous atoms are clamped by energy knots of outer rings belonging to solid atoms. This is done under the suitable employed conditions. Here, energy is involved to engage the forced behaviours in surface format for transitional behaviors gaseous and solid atoms. However, this is under the maintenance of midpoint of gaseous and solid atoms in the surface format.

In $\mathrm{Ti}$ atom, filled state electrons deal with the maximum gravitational force. So, they also possess the maximum expansion of clamped energy knots. Thus, the electrons of $\mathrm{Ti}$ atoms keep the original ground point below the suitable level of ground surface. $\mathrm{N}$ atom belongs to gaseous state that keeps ground point above the suitable level of ground surface where electrons experience the maximum levitational force. This way, electrons of $\mathrm{N}$ atom possess the maximum contraction of clamped energy knots.

The electrons of $\mathrm{N}$ atoms undertake double clamps of energy knots of $\mathrm{Ti}$ atoms, where tips of electrons experience the force through emptiness of unfilled states. So, 
the pieces of non-conserved energy (representing the heat energy) provide the paths to suitable electrons of $\mathrm{N}$ atoms to undertake double clamps of energy knots of $\mathrm{Ti}$ atoms. This is done when gaseous atoms attempt to leave the surface format and solid atoms attempt to enter the grounded format in the course of transitional behaviours. So, both gaseous and solid natured atoms accommodate their ground points at substrate surface or just above the substrate surface. In this manner, they adhere to deposit at the surface of substrate. The mechanism of the double clamping of energy knots (of $\mathrm{Ti}$ atoms) to electrons (of $\mathrm{N}$ atoms) is shown in Figure 6. Here, bound $\mathrm{N}$ atom to Ti atom is adjusted at interstitial position developed by four $\mathrm{Ti}$ atoms as shown in Figure 6 . A suitable electron of landing $\mathrm{Ti}$ atom (in less expansion) adheres to already landed $\mathrm{Ti}$ atom (in more expansion). They adhere through the double clamping of energy knot to suitable electron. So, a Ti atom binds to another Ti-atom, which is shown in Figure 6.

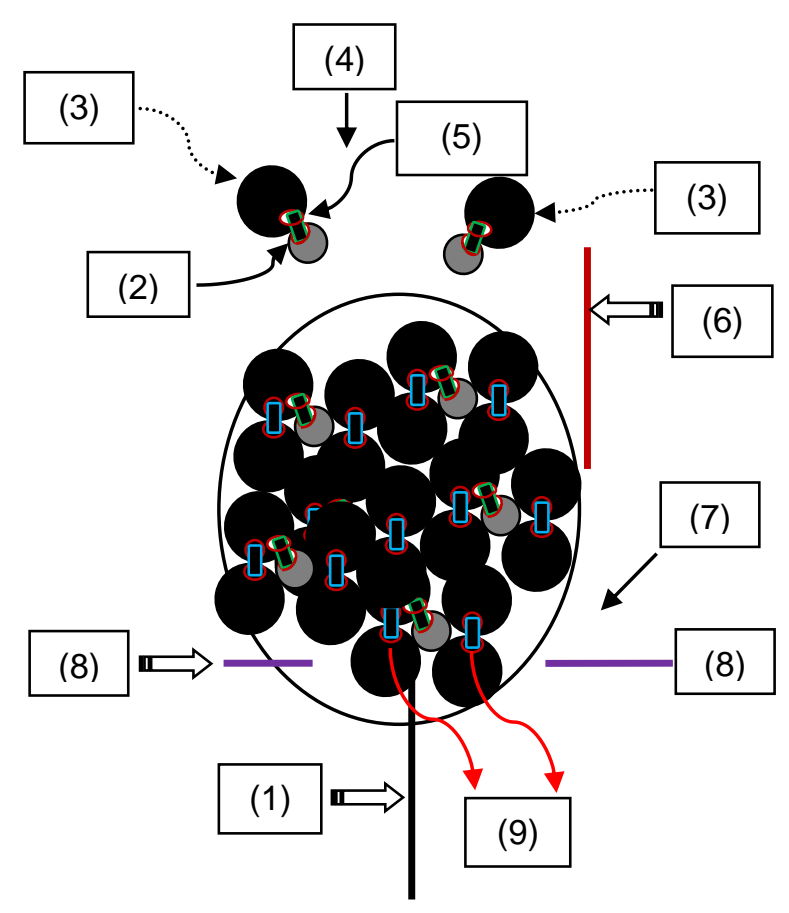

Figure 6: Mechanisms of double clamping of energy knots (of solid atoms) to electrons (of gaseous atoms) and binding Ti-Ti atoms; (1) solid atoms retain ground point below the suitable level of ground surface, (2) suitable electron of $N$ atom just attempted to recover original state, (3) an atom ejected from Ti target, (4) inlet of $\mathrm{N}$ gas, (5) suitable energy knot of Ti atom just attempted to recover original state, (6) gaseous atoms retain ground point above the suitable level of ground surface, (7) HSS disc, (8) solid and gaseous atoms attained ground points at or above the substrate surface and (9) Ti-Ti binding 
Under the action of tailored energy of $\mathrm{N}$ atoms and $\mathrm{Ti}$ atoms, they react and develop affinity in the form of TiN coating at the substrate surface. Solid atoms in original state keep ground points in grounded format. So in solid atoms, electrons possess the maximum potential energy, clamped energy knots to electrons are in their maximum expansion and electrons deal with the maximum orientating levitational force along the relevant poles. Electrons fully deal with gravitational behaviour of exerting force along the relevant poles. Gaseous atoms in original state keep ground points in space format. So in gaseous atoms, electrons possess the minimum potential energy, clamped energy knots to electrons are in their maximum contraction and electrons deal with the maximum orientating levitational force along the relevant poles.

When a $\mathrm{Ti}$ atom lands at substrate, it attempts to recover original solid state, where electrons gravitate. However, prior to being fully gravitated, an adequate expansion of 'energy knot net' takes place, which is under the adjustable potential energy of electrons. Ti atom attains ground point on the surface of previously landed $\mathrm{Ti}$ atom due to the less expansion of energy knot net. This way, a certain electron of less expanded landed $\mathrm{Ti}$ atom is being forced to experience the exerting force through the emptiness of suitable unfilled energy knot of a more expanded previously landed $\mathrm{Ti}$ atom. Both $\mathrm{Ti}$ atoms adjust expansion/contraction to tighten energy knot (of already landed $\mathrm{Ti}$ atom) clamped to electron (of just landed Ti atom). Thus, a bit difference in their ground points results in the binding. $\mathrm{N}$ atoms attempt to recover state to go into original gas state; their suitable electrons experience force through the emptiness of suitable unfilled energy knots of Ti atoms. This is from the rear side of solid atom. These binding mechanisms of two different natured atoms ( $\mathrm{Ti}-\mathrm{N}$ ) and the same natured atoms ( $\mathrm{Ti}-\mathrm{Ti})$ provide the space for $\mathrm{N}$ atoms to be at the interstitial positions. In gaseous atoms, involved energy is inversely proportional to the engaged force, whereas involved energy is directly proportional to the engaged force in solid atoms [4].

When solid atom is just recovering from the transition state, it allocates certain unfilled energy knot belonging to outer ring to take another clamp for certain filled state electron of gaseous atom, which is also recovering from the certain transition state. The reaction of gaseous atom occurs when ground point is at the level of exerting force in 
surface format instead of at the level of exerting force in space format. The reaction of metallic atom occurs when ground point is at the level of exerting force in surface format instead of at the level of exerting force in grounded format. This results in the binding of different atoms at a common ground point. The common ground point of reaction is at the mid-ground points of gaseous atom exerting force to electrons originally in space format and solid atom exerting force to electrons originally in grounded format. The binding of gaseous and solid atoms under attained transition states engages the forces of both upward (space) and downward (grounded) formats as per involved energy. Hence, their structure gets hard. So, electrons in different natured atoms adjust the expansion and contraction of clamped energy knots. Here, energy is being involved and force is being engaged. Both are in the non-conservative mode.

A low measured surface roughness in $\mathrm{CrN}$ coating (than $\mathrm{TiN}$ coating) is given elsewhere [25]. So, a low measured hardness of $\mathrm{CrN}$ coating than $\mathrm{TiN}$ coating can be due to a bit varied mechanism of binding in their different natured atoms. The binding mechanism in $\mathrm{CrN}$ requires further clarifications. High probability of binding is involved as $\mathrm{Cr}$ atom contains many unfilled states for the outer ring as compared to $\mathrm{Ti}$ atom. The electron of outer ring belonging to gaseous atom ( $\mathrm{N}$ atom) reacts developing $\mathrm{CrN}$ by having another clamp of energy knot belonging to outer ring in $\mathrm{Cr}$ atom. At the time of recovering transition state, $\mathrm{Cr}$ atoms are just at the substrate surface, so they react with $\mathrm{N}$ atoms under its recovering transition state, too. A landing $\mathrm{Cr}$ atom binds to landed $\mathrm{Cr}$ atom under the difference of their expansion. In this manner, atoms devise the unit (primitive) cell of hard coating. $\mathrm{N}$ atom is also incorporated at interstitial position of $\mathrm{Cr}$ atoms. A similar approach may be considered to explore the science of other hard, moderate hard and even less hard materials. A bit different mechanism can be anticipated in binding of bi-metallic composition (atoms), for example, in TiAIN coating. A quite different mechanism may be anticipated in the case of TiCN coating because of the involvement of carbon atom. This requires further studies to express the science of binding different atoms in TiCN coating.

Conservative forces are involved to engage energy in the form of forcing energy (travelling photon), where a photon having characteristics of current is discussed [1]. 
Whenever the element of force is involved, the energy is engaged as in the case of silicon atom [2]. However, developing hard coating is due to the involvement of nonconservative energy, where non-conservative force is engaged at later stage.

Formation of hard coating does not indicate the roles of gravitational and levitational forces apparently. However in binding atoms, electrons retain states by adjusting and maintaining infinitesimal displacements at required level. An involved energy engages the force to achieve required infinitesimal displacements of the electrons. In the deposition chamber, randomly distributed arc spotting at the target to eject metallic atoms involved both energy and force. Entitled energy for required transition states of the atoms also engages the components of force at the instant of their binding. A process of transitional energy regulates the structure of hard coating under engaging forces of atoms at electron levels. The process energy maintains the required transition states of gaseous and solid atoms through varying the potential energy of electrons. For required transition state of solid atom, the potential energy of electrons was decreased. (Thus, potential energy of electrons controlled by orientating gravitational force). For required transition state of gaseous atom, the potential energy of electrons was increased. (Thus, potential energy of electrons controlled by orientating levitational force). A further detail of energy and forced behaviours in solid atom and gaseous atoms can be found elsewhere [4].

Suitable gaseous and solid atoms under certain process parameters develop hard coating. Different atoms bind by double clamping of energy knots to electrons. Appropriate vacuum conditions and high power increase the hardness of a coating. Hard coatings develop with different characteristics because of having a variety of structures under varied energy and force. The lifetime of a hard coating depends on the developing strategy. This way, they directly or indirectly explore many new areas of modern research.

\section{Conclusion}

In random arc-based vapor deposition, TiN coating deposits on high-speed steel tool on just next to the Ti inter-layer. So, incorporated metallic inter-layer improves the adhesive 
strength of followed TiN coating. Fracture cross-sectional image also infers the detail of compact and dense TiN coating just following the inter-layer. The elemental compositions of $\mathrm{Ti}$ and $\mathrm{N}$ indicate a uniform distribution as examined by the macro droplet of coating at the surface. In TiN coating, about $70 \%$ content is related to $\mathrm{Ti}$ atoms and about $30 \%$ content is related to $\mathrm{N}$ atoms. The presence of gaseous atoms with solid atoms introduces insulating behaviour of hard coating.

In the mechanism of developing hard coating, a gaseous atom of suitable transition state partially hands over the electron of outer ring to energy knot of outer ring of solid atom when also in suitable transition state. Gaseous atom binds to solid atom from the rear side in the attempt to restore original state. Due to locating ground point of gaseous atom in the surface format under suitable transition state, it binds to solid atom coming downwardly to deposit at the substrate surface. Here, solid atom was coming to restore the original state in the grounded format of exerting force. However, in the deposition chamber, a substrate is placed below it.

In developing hard coating, gaseous atoms react to solid atoms when in an attempt to recover their transition states. A same is the case with solid atoms. So, in this adventure, different natured atoms appropriately coincide to bind as their established transition states favour the binding. Here, a suitable electron of gaseous atom experiences the force to undergo for another clamp of energy knot belonging to a suitable unfilled state of solid atom. Different natured atoms adjust forced behaviour through involved heat energy of the process. So, atoms position electrons in the orientations required for their binding under the involvement of energy and engagement of force at electron level. Both energy and force obey the non-conservation.

At common ground point, an electron of gaseous atom experiences force to northsided tip through emptiness of a suitable unfilled energy knot of solid atom. That electron of gaseous atom increases potential energy by orientating under decreasing levitational force. An energy knot of unfilled state positions because of decreasing potential energy of electrons in its solid atom under the lowering orientating gravitational force. In an atom of transition state, orientation of the electron is being controlled by the involved heat energy [4]. 
Under suitable coinciding, an energy knot of outer ring in solid atom clamps to electron of outer ring in gaseous atom. Expansion and contraction of gaseous atom and solid atom adjusts accordingly. So, hard coating is deposited because of incompatible working energy and forced behaviours of different natured atoms. A Ti atom binds with another $\mathrm{Ti}$ atom under the difference of their expansions. $\mathrm{N}$ atoms grow under the different scheme of electronic structure when deal with the force mainly in space format. $\mathrm{Ti}$ atoms grow under the scheme of exerting forces in the grounded format. Gaseous atoms at interstitial positions of $\mathrm{Ti}$ atoms deteriorate inter-state electron gaps of metallic nature atoms. So, TiN coating works for insulating behaviour both in terms heat energy and photon energy.

To deposit TiN coating on a suitable substrate, energy and forced behaviours of transitional gaseous and solid atoms work in different manners. Certain transition metals govern hard features of coating as a result of their strong affinity to gaseous atoms because one is originally related to exerting force (to electrons) in grounded format and the other is originally related to exerting force (to electrons) in space format. However, under desirable switching of energy and forced behaviours, atoms of different natures attain ground points nearly at the midpoint of exerting forces in the grounded format and space format. So, involved heat energy of the process is utilized to adjust the forced behaviours of different natured atoms regulating conditions for surface format environment. However, in this attempt, a structure of hard coating do not deal with a consistent photonic band gap.

This fundamental study describing the developmental mechanism of hard coating suggests the ways and means to develop smart deposition chamber systems, where controlling pressure and temperature along with other parameters in the appreciable ranges can maintain high precisions. This is very much possible through the automation in addition to semi-automation of deposition systems. This study suggests briefly the kind of component that is required to obtain planned results of material properties and their characteristics. This will help achieve the unique features of coatings and their meaningful utilization. 
Controlling the lateral and adjacent orientation of electrons for depositing atoms at interface and final layer stage (surface) respectively will result in the unprecedented performance of tools. When the application of such coating is for an ultra-precision machining, it can deliver high-performance in the case of dry machining. Such strategies will not only save the revenue but also keep the environment clean.

\section{Acknowledgements:}

Mubarak Ali is obliged to Malaysian Government for awarding scholar of PhD study (2004-07) under award letter No. JPA(L) KD333487 and Government of Pakistan (Pakistan Council of Renewable Energy Technologies (PCRET), Ministry of Science \& Technology) for granting the study leave. Dr. Mubarak greatly appreciates the kind support of Ex-Director Generals, PCRET, Dr. Parvez Akhter and Professor Dr. Ishtiaq A. Qazi. He is also grateful to Malaysian and International colleagues at AMREC, SIRIM Berhad and at UTM Johor for their kind hospitality. Mubarak also thanks Professor Dr. Ali Ourdjini to review the work at several occasions and for constructive feedback while studying at UTM.

\section{References}

[1] M. Ali, Atoms of None of the Elements lonize While Atoms of Inert Behavior Split by Photonic Current. (2020), http://arxiv.org/abs/1611.05392 (last version)

[2] M. Ali, Heat and Photon Energy Phenomena: Dealing with Matter at Atomic and Electronic Level. (2017), https://www.preprints.org/manuscript/201701.0028 (last version)

[3] M. Ali, Structure Evolutions in Atoms of Those Elements Executing Confined Inter-State Electron Dynamics. (2020), http://arxiv.org/abs/1611.01255 (last version)

[4] M. Ali, Atoms in Gas and Solid States and their Energy and Force Relationships under Transitional Behaviors. (2020), https://www.researchgate.net/publication/323723379 (last version) 
[5] M. Ali, Atomic Structure and Binding of Carbon Atoms. (2019), https://www.preprints.org/manuscript/201801.0036 (last version)

[6] M. Ali, Nanoparticles-Photons: Effective or Defective Nanomedicine, J. Nanomed. Res. 5 (2018) 241-243.

[7] S. C. Glotzer, M. J. Solomon, Anisotropy of building blocks and their assembly into complex structures, Nature Mater. 6 (2007) 557-562.

[8] S. Link, M. A. El-Sayed, Shape and size dependence of radiative, nonradiative and photothermal properties of gold nanocrystals, Int. Rev. Phys. Chem. 19 (2000) 409- 453.

[9] M. Ali, I $-\mathrm{N}$. Lin, Formation of tiny particles and their extended shapes: origin of physics and chemistry of materials, Appl. Nanosci. 9 (2019) 1367-1382.

[10] M. Ali, I -N. Lin, C. -J. Yeh, Tapping Opportunity of Tiny-Shaped Particles and Role of Precursor in Developing Shaped Particles, NANO 13 (7) (2018) 1850073 (16 pages).

[11] M. Ali, I -N. Lin, C. -J. Yeh, Predictor Packing in Developing Unprecedented Shaped Colloidal Particles, NANO 13 (9) (2018) 1850109 (15 pages).

[12] M. Ali, I $-\mathrm{N}$. Lin, Phase transitions and critical phenomena of tiny grains carbon films synthesized in microwave-based vapor deposition system, Surf. Interface Anal. 51 (2019) 389-399.

[13] M. Ali, M. Ürgen, Switching dynamics of morphology-structure in chemically deposited carbon films -A new insight, Carbon 122 (2017) 653-663.

[14] M. Ali, M. Ürgen, Morphology and Structure of Carbon Films Deposited at Varying Chamber Pressures. (2020), https://arxiv.org/abs/1802.00730 (last version)

[15] H. Wroe, The magnetic stabilization of low pressure d.c. arcs, British J. Appl. Phys. 9 (1958) 488-491.

[16] R. L. Boxman, D. M. Sanders, and P. J. Martin (editors), Handbook of Vacuum Arc Science and Technology; Fundamentals and applications, Noyes Publications, New Jersey, Park Ridge (1995). 
[17] D. A. Karpov, Cathodic arc sources and macroparticle filtering, Surf. Coat. Technol. 96 (1997) 22-33.

[18] I. G. Brown, Cathodic arc deposition of films, Annu. Rev. Mat. Sci. 28 (1998) 243269.

[19] M. Ali, E. Hamzah, M. R. M. Toff, A. H. Hashim, The effect of Nitrogen gas flow rate on the properties of TiN-coated HSS using Cathodic Arc Evaporation PVD Technique, Surf. Rev. Lett. 12 (2005) 631-643.

[20] E. Hamzah, A. Ourdjini, M. Ali, P. Akhter, M. R. M. Toff, M. A. Hamid, Influence of nitrogen flow rate on friction coefficient and surface roughness of TiN coatings deposited on tool steel using arc method, Surf. Rev. Lett. 14 (2007) 1007-1013.

[21] M. Ali, P. Akhter, E. Hamzah, M. R. M. Toff, I. A. Qazi, Effect of coating thickness on the properties of TiN coatings deposited on tool steels using cathodic arc PVD technique, Surf. Rev. Lett. 15 (2008) 401-410.

[22] M. Ali, E. Hamzah, M. R. M. Toff, Friction coefficient and surface roughness of TiN-coated HSS deposited using cathodic arc evaporation PVD technique, Ind. Lubr. Tribol. 60 (2008) 121-130.

[23] M. Ali, E. Hamzah, T. Abbas, M. R. M. Toff, 1. A. Qazi, Macrodroplet reduction and growth mechanisms in cathodic arc physical vapour deposition of TiN films, Surf. Rev. Lett. 15 (2008) 653-659.

[24] M. Ali, E. Hamzah, I. A. Qazi, M. R. M. Toff, Effect of Cathodic Arc PVD Parameters on Roughness of TiN Coating on Steel Substrate, Curr. Appl. Phys. 10 (2010) 471-474.

[25] M. Ali, E. Hamzah, M. R. M. Toff, Study of macrodroplet and growth mechanisms with and without ion etchings on the properties of TiN coatings deposited on HSS using cathodic arc physical vapour deposition technique, Mater. Sci. Eng. A 474 (2008) 236-242.

[26] H. Elmkhah, F. Attarzadeh, A. Fattah-alhosseini, K. H. Kim. Microstructural and electrochemical comparison between TiN coatings deposited through HIPIMS and DCMS techniques, J. Alloy. Compd. 735 (2018) 422-429. 
[27] J. C. Oliveiraa, F. Fernandesa, R. Serraa, A. Cavaleiro. On the role of the energetic species in TiN thin film growth by reactive deep oscillation magnetron sputtering in $\mathrm{Ar} / \mathrm{N}_{2}$, Thin Solid Films 645 (2018) 253-264.

[28] U. Vogel, S. Oswalda, J. Eckert. Interface and stability analysis of Tantalum- and Titanium nitride thin films onto Lithiumniobate, Appl. Surf. Sci. 425 (2017) 254260.

[29] G. T. P. Azar, C. Yelkarasi, M. Ürgen, The role of droplets on the cavitation erosion damage of TiN coatings produced with cathodic arc physical vapor deposition, Surf. Coat. Technol. 322 (2017) 211-217.

[30] A. Bahri, E. Kaçar, S. S. Akkaya, K. Elleuch, M. Ürgen, Wear protection potential of TiN coatings for 304 stainless steels used in rotating parts during olive oil extraction, Surf. Coat. Technol. 304 (2016) 560-566.

[31] M. F. Othman, A. R. Bushroa, W. N. R. Abdullah. Evaluation techniques and improvements of adhesion strength for TiN coating in tool applications: a review, J. Adhes. Sci. Technol. 29 (2015) 569-591.

[32] Q. W. Jiang, G. R. Li, X. P. Gao, Highly ordered TiN nanotube arrays as counter electrodes for dye-sensitized solar cells, Chem. Commun. 44 (2009) 6720-6722.

[33] S. G. Harris, E. D. Doyle, Y. -C. Wong, P. R. Munroe, J. M. Cairney, J. M. Long. Reducing the macroparticle content of cathodic arc evaporated TiN coatings, Surf. Coat. Technol. 183 (2004) 283-294.

[34] L. von Fieandta, K. Johanssona, T. Larssonb, M. Bomana, E. Lindahlc, On the growth, orientation and hardness of chemical vapor deposited $\mathrm{Ti}(\mathrm{C}, \mathrm{N})$, Thin Solid Films 645 (2018) 19-26.

[35] K. Bobzin, High-performance coatings for cutting tools, CIRP Jour. Manuf. Sci. Technol. 18 (2017) 1-9.

[36] Q. Wang, F. Zhou, Progress in Tribological Properties of Nano-Composite Hard Coatings under Water Lubrication, Lubricants 5 (2017) 5.

[37] D. Music, R. W. Geyer, J. M. Schneider, Recent progress and new directions in density functional theory based design of hard coatings, Surf. Coat. Technol. 286 (2016) 178-190. 
[38] E. Santecchiaa, A. M. S. Hamoudaa, F. Musharavatia, E. Zalnezhadb, M. Cabibboc, S. Spigarellic, Wear resistance investigation of titanium nitride-based coatings, Ceram. Int. 41 (2015) 10349-10379.

[39] Y. X. Wang, S. Zhang, Toward hard yet tough ceramic coatings, Surf. Coat. Technol. 258 (2014) 1-16.

[40] A. Inspektor, P. A. Salvador, Architecture of PVD coatings for metal cutting applications: A review, Surf. Coat. Technol. 257 (2014) 138-153.

[41] A. D. Pogrebnjaka, A. V. Pshyka, V. M. Beresnevb, B. R. Zhollybekova, Protection of specimens against friction and wear using titanium-based multicomponent nanocomposite coatings: A review, J. Frict. Wear 35 (2014) 5566.

[42] M. Fenker, M. Balzer, H. Kappl, Corrosion protection with hard coatings on steel: Past approaches and current research efforts, Surf. Coat. Technol. 257 (2014) 182-205.

[43] Y. Purandarea, A. Ehiasarian, A. Santana, P. Hovsepian, ZrN coatings deposited by high power impulse magnetron sputtering and cathodic arc techniques, J. Vac. Sci. Technol. A 32 (2014) 031507.

[44] C. Mitterer, F. Holler, F. Üstel, D. Heim, Application of hard coatings in aluminium die casting-soldering, erosion and thermal fatigue behaviour, Surf. Coat. Technol. 125 (2000) 233-239.

[45] W. -D. Münz, T. Hurkmans, G. Keiren, T. Trinh, Comparison of TiAIN coatings grown by unbalanced magnetron and arc bond sputtering techniques, J. Vac. Sci. Technol. A 11 (1993) 2583-2589.

[46] W. Tanglumlert, P. Prasassarakich, P. Supaphol, S. Wongkasemjit, Hard-coating materials for poly(methyl methacrylate) from glycidoxypropyltrimethoxysilanemodified silatrane via a sol-gel process, Surf. Coat. Technol. 200 (2006) 27842790.

[47] Soni, S. Kumari, S. K. Sharma, S. K. Mishra, Effect of Deposition Pressure, Nitrogen Content and Substrate Temperature on Optical and Mechanical 
Behavior of Nanocomposite Al-Si-N Hard Coatings for Solar Thermal Applications, J. Mater. Eng. Perform. 27 (2018) 6729-6736.

[48] O. A. Fouad, H. - Y Lin, S. I. Shah, Reactively Magnetron Sputter-Deposited Ti (C, N) Nanocomposite Thin Films: Composition and Thermal Stability, J. Coat. Sci. Technol. 5 (2018) 42-49.

[49] A. Eshaghi, Transparent hard self-cleaning nano-hybrid coating on polymeric substrate, Prog. Organic Coat. 128 (2019) 120-126.

[50] J. H. Huang, Y. F. Chen, G. P. Yu, Evaluation of the fracture toughness of $\mathrm{Ti}_{1}$ $x Z r_{x} N$ hard coatings: Effect of compositions, Surf. Coat. Technol. 358 (2019) 487496.

[51] P. C. Siow, J. A. Ghani, M. Rizal, T. R. Jaafar, M. J. Ghazali, C. H. Che Haron, Surface characterisation of $\mathrm{TiC}_{\times} \mathrm{N}_{1-\mathrm{x}}$ coatings processed by cathodic arc physical vapour deposition: XPS and XRD analysis, Surf. Interface Anal. 51 (2019) 611 617.

[52] G. M. Uddin, et al., Experimental investigation of tribo-mechanical and chemical properties of TiN PVD coating on titanium substrate for biomedical implants manufacturing, Int. J. Adv. Manuf. Technol. 102 (2019) 1391-1404.

[53] A. Dehghanghadikolaei, B. Fotovvati, Coating Techniques for Functional Enhancement of Metal Implants for Bone Replacement: A Review, Materials, 12 (2019) 1795.

[54] B. Fotovvati, N. Namdari, A. Dehghanghadikolaei, On coating techniques for surface protection: A review, J. Manufacturing and Materials Processing. 3 (2019) 28. 


\section{Authors' biography:}

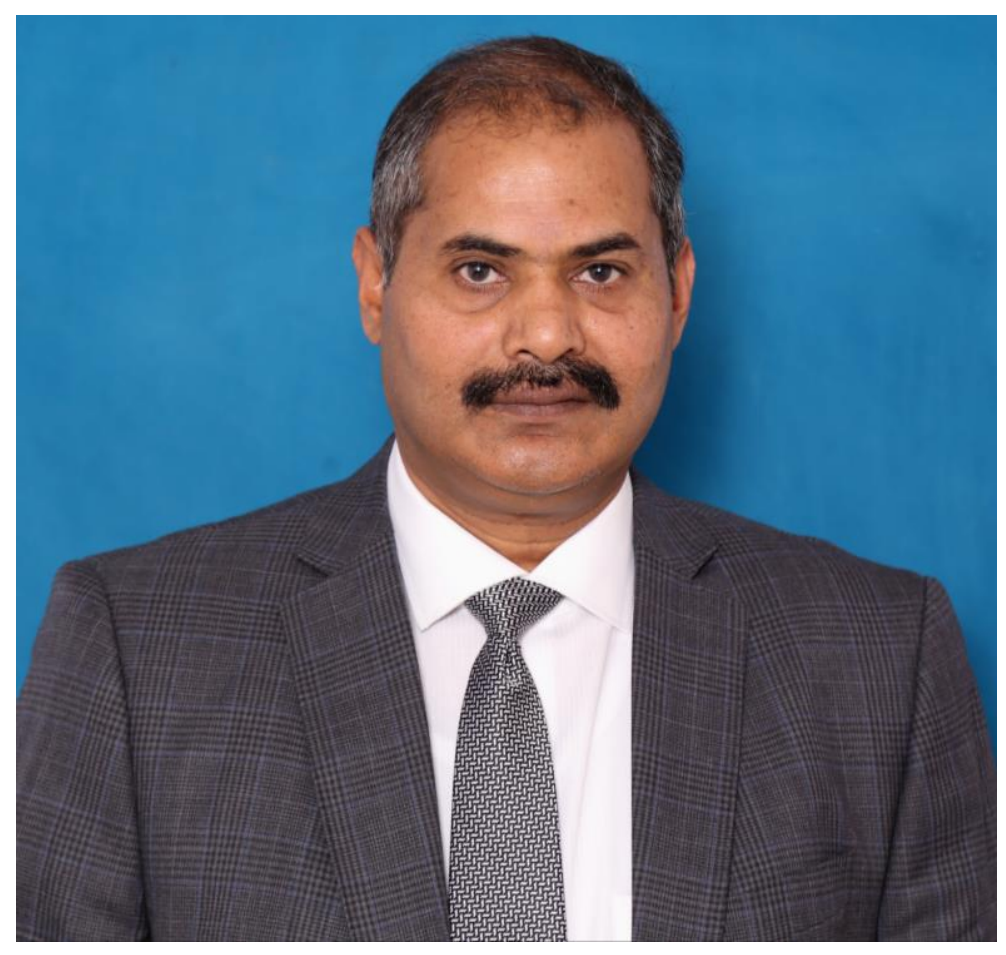

Mubarak Ali graduated from University of the Punjab with BSc (Phys \& Maths) in 1996 and MSc Materials Science with distinction from Bahauddin Zakariya University, Multan, Pakistan (1998); his thesis work completed at Quaidi-Azam University Islamabad. He gained PhD in Mechanical Engineering from the Universiti Teknologi Malaysia under the award of Malaysian Technical Cooperation Programme (MTCP;2004-07) and postdoc in advanced surface technologies at Istanbul Technical University under the foreign fellowship of The Scientific and Technological Research Council of Turkey (TÜBITAK, 2010). He completed another postdoc in the field of nanotechnology at the Tamkang University Taipei (2013-2014) sponsored by National Science Council now M/o Science and Technology, Taiwan (R.O.C.). Presently, he is working as Assistant Professor on tenure track at COMSATS University Islamabad (previously known as COMSATS Institute of Information Technology), Islamabad, Pakistan (since May 2008) and prior to that worked as assistant director/deputy director at M/o Science \& Technology (Pakistan Council of Renewable Energy Technologies, Islamabad, 2000-2008). He was invited by Institute for Materials Research, Tohoku University, Japan to deliver scientific talk. He gave several scientific talks in various countries. His core area of research includes materials science, physics \& nanotechnology. He was also offered the merit scholarship for the PhD study by the Higher Education Commission, Government of Pakistan, but he did not avail himself of the opportunity. He also earned Diploma (in English language) and Certificate (in Japanese language) in 2000 and 2001 respectively, in part-time from the National University of Modern Languages, Islamabad. He is the author of several articles available at following links;

https://scholar.google.com.pk/citations?hl=en\&user=UYjuhDwAAAAJ, https://www.researchgate.net/profile/Mubarak Ali5, https://www.mendeley.com/profiles/mubarak-ali7/, \& https://publons.com/researcher/2885742/mubarak-ali/publications/ 


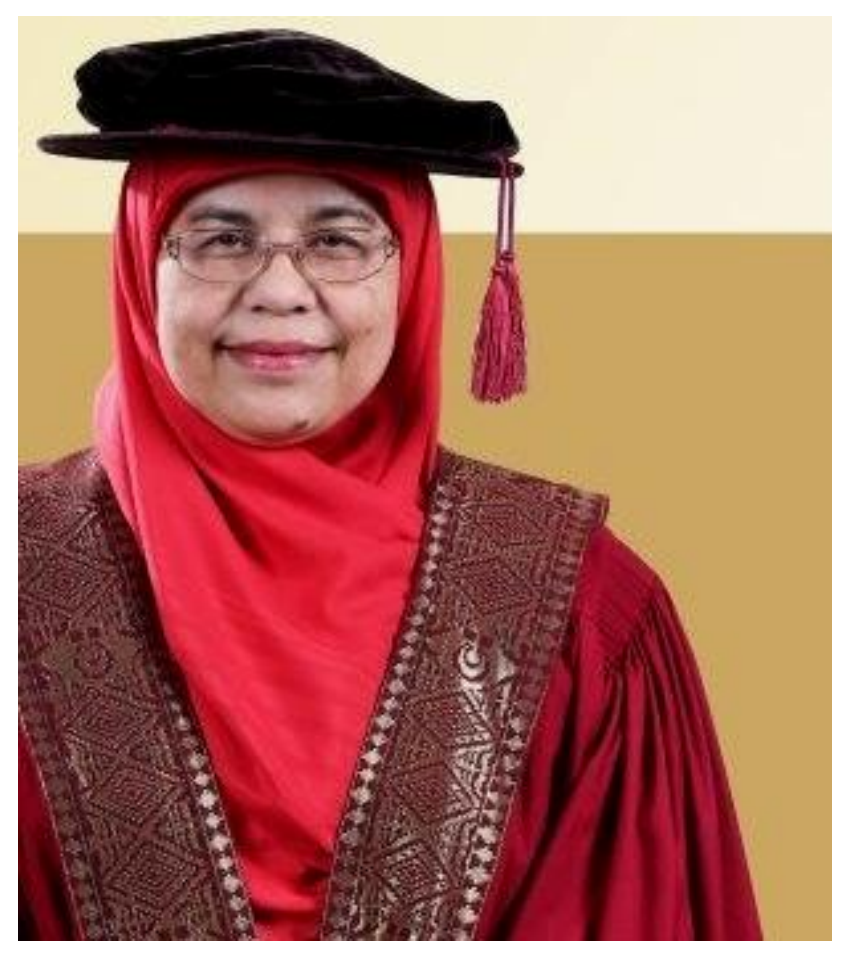

Dr Esah Hamzah is a senior standing Professor at the Universiti Teknologi Malaysia in the Faculty of Mechanical Engineering. Dr Esah obtained her BSc degree from the University of Wales at Swansea, UK, and MSc and PhD in Metallurgy from the University of Manchester Institute of Science and Technology (U.M.I.S.T.), UK. She has over 30 years of experience in teaching the undergraduate and postgraduate courses related to metallurgy and materials engineering. She has also supervised both masters and PhD students and has served as an examiner for MSc and PhD theses. She has held various administrative positions at the Universiti Teknologi Malaysia and remained Head of Department (1999-2005) and Deputy Dean (Academics) of the Faculty of Mechanical Engineering (2005-2010). She has been actively involved in research in the areas of phase transformation and mechanical behavior of metals, metal failure, corrosion and coating. She has been awarded many research grants from the University and the Malaysian Government. She has also presented many papers in the national/international conferences and some of her papers won the "Best Paper" award. She is also a contributor to the University's Best Publication Award won by the Faculty of Mechanical Engineering in series of years along with Excellent Service for the year 1993 and the University Excellence Award in 2000, 2003 and 2007. Dr Esah is an active Council Member, charted engineer, and a pioneering Fellow member of the Institute of Materials Malaysia (IMM). She is the author of several articles available at the following links;

https://scholar.google.com.pk/citations?user=YHkEpzYAAAAJ\&hl=en and https://www.researchgate.net/profile/Esah_Hamzah

Dr. Mohd Radzi Mohd Toff is a General Manager at Advanced Materials Research Centre (AMREC), SIRIM BHD, Malaysia and prior to that worked as head of coating technology group at AMREC and principal scientist. He graduated from the Universiti Kebangsaan Malaysian (UKM) and earned PhD in Chemical Engineering from The University of Sheffield, UK. 